\title{
Germanica
}

\section{Max Reinhardt, figure budapestoise}

Max Reinhardt, eine Gestalt auf der Budapester Szene

\section{Katalin Pór}

\section{OpenEdition}

\section{Journals}

Édition électronique

URL : http://journals.openedition.org/germanica/579

DOI : 10.4000/germanica.579

ISSN : 2107-0784

Éditeur

Université de Lille

\section{Édition imprimée}

Date de publication : 1 décembre 2008

Pagination : 151-160

ISBN : 978-2-913857-22-6

ISSN : 0984-2632

\section{Référence électronique}

Katalin Pór, « Max Reinhardt, figure budapestoise », Germanica [En ligne], 43 | 2008, mis en ligne le 01 décembre 2010, consulté le 06 octobre 2020. URL : http://journals.openedition.org/germanica/579 ; DOI : https://doi.org/10.4000/germanica.579

Ce document a été généré automatiquement le 6 octobre 2020.

(C) Tous droits réservés 


\title{
Max Reinhardt, figure budapestoise
}

\author{
Max Reinhardt, eine Gestalt auf der Budapester Szene
}

Katalin Pór

1 Les histoires de la littérature hongroise n'accordent le plus souvent qu'une portion congrue à sa production théatrale. La Hongrie, sans doute du fait de la multiplicité sur son sol de théatres en langue allemande - du moins jusqu'en 1889, date à laquelle le dernier théâtre en allemand ferme - n'est pas véritablement parvenue à instaurer une tradition théâtrale durable. Si la situation évolue au tournant du siècle, avec la prolifération à Budapest de lieux de représentation et le développement d'une véritable industrie théâtrale, cette production ne s'inscrit que marginalement dans la création artistique nationale. On peut notamment percevoir une forte cohérence entre les productions poétiques et romanesques; il en va différemment de la création théâtrale, qui semble relativement déconnectée de ces recherches.

2 Elle s'inscrit en revanche très clairement dans le système de circulations théâtrales européennes, qui connaît à cette période une amplification considérable ${ }^{1}$. Les mouvements de correspondance, de transferts, d'échanges entre les institutions théâtrales budapestoises et celles du reste de l'Europe, déjà présents à la fin du XIXe , se trouvent en effet renforcés par l'implantation au début du $\mathrm{XIX}^{\mathrm{e}}$ dans la capitale hongroise de nouvelles institutions théâtrales, se voulant plus modernes, plus ouvertes sur le monde extérieur et plus propices à intégrer des innovations. Ainsi, la création du Théâtre Comique, en 1896, celle du Théâtre Hongrois, qui ouvre l'année suivante, suivie par le Théâtre du Roi en 1903 et le Théâtre Populaire en 1911 permettent une véritable accélération des échanges.

3 Parmi les différentes contributions extérieures, ce sont les apports germanophones (allemands et autrichiens) qui dominent, tant par la quantité de spectacles importés que par l'importance qui leur est accordée au sein de la vie intellectuelle hongroise. Cet état de fait n'a rien de surprenant et s'explique évidemment par la forte proximité entre la Hongrie et l'espace germanophone. Ainsi, les passages de Max Reinhardt, dans la continuité de ceux d'Otto Brahm, marquent profondément le théâtre hongrois : ils 
sont l'objet de phénomènes conjoints de starification et d'appropriation, qui imposent Reinhardt comme une figure centrale de la vie théâtrale budapestoise.

\section{D’Otto Brahm à Max Reinhardt}

4 Le phénomène des compagnies invitées, qui se systématise en Hongrie au tournant du siècle, joue un rôle primordial dans l'importation des innovations étrangères. Les nouveaux théatres y ont en effet massivement recours, d'abord pour combler les trous de la programmation, puis en tant qu'événement artistique à part entière. Ce mouvement permet tout d'abord l'introduction du naturalisme. Dans de nombreux articles $^{2}$,Géza Staud, historien du théâtre hongrois, insiste en effet sur l'importance de l'arrivée du naturalismeen Hongrie dans les dernières années du XIX ${ }^{e}$ siècle. Il décrit une série de vagues successives; le public hongrois serait d'abord initié par l'arrivée des Meiningen et de leur historicisme ${ }^{3}$ : ceux-ci se rendent à quatre reprises en Hongrie, en $1875,1879,1881$ et 1888 . Ce sont ensuite les naturalistes italiens qui viennent se produire. Staud cite notamment Ermete Zacconi, Gustavo Salvini, et Eleonore Duse, qui apportent un jeu d'acteur basé sur la suggestivité. Enfin, en 1901, Otto Brahm et son Deutsches Theater de Berlin se produisent pour la première fois dans la capitale hongroise : ces représentations ont un retentissement considérable et une influence durable, en faisant la démonstration de l'importance de la mise en scène dans la représentation. Otto Brahm continue ensuite à venir très régulièrement avec le Deutsches Theater en 1901, 1902 et 1903, puis avec le Lessing Theater en 1905. Staud évoque également des influences françaises, notamment le Théâtre Libre d'Antoine: cette influence est cependant plus indirecte et arrive essentiellement par le biais d'otto Brahm. L'apport naturaliste ne saurait être réduit aux passages de Brahm à Budapest : sa figure va toutefois progressivement concentrer ce type d'apport, et fonctionner à la fois comme un vecteur et un emblème de celui-ci.

Quant à Reinhardt, il se produit d'abord en Hongrie en tant qu'acteur : ses premières visites datent de 1893. La venue d'acteurs de langue allemande à Budapest est alors un non-événement; si ses apparitions sont signalées dans plusieurs journaux germanophones, le public budapestois n'y prête pas spécialement attention. Ces mêmes journaux commencent cependant à s'intéresser à lui en 1899, tandis que le Berliner Ensemble vient jouer à Budapest un programme composé de pièces de Hauptmann, de Wolzogen et de Tolstoï. Non seulement le spectacle obtient un succès considérable, mais la performance d'acteur de Reinhardt y est unanimement saluée. Encouragée par ce succès, la compagnie revient l'année suivante et reçoit le même accueil. Otto Brahm signe alors un contrat avec le Théâtre de la Comédiehongrois et vient y jouer dix pièces l'année suivante, en avril 1901. Le succès ne se dément pas et Otto Brahm revient une année de plus. Max Reinhardt, dont les prestations sont toujours aussi remarquées, se rend en 1903 à Budapest sans Brahm avec son cabaret, le Schall und Rauch : le succès est colossal, notamment parce que le cabaret est alors un genre en pleine crise à Budapest.

En 1903, puis en 1904, Reinhardt revient pour la première fois en tant que directeur de compagnie, avec le Kleines und Neues Theater: il est alors à la fois metteur en scène, directeur et acteur. Si la critique note son talent de metteur en scène, c'est cependant toujours comme acteur qu'il est avant tout reconnu et apprécié. La situation change dans les deux années qui suivent : Reinhardt ne retourne plus en Hongrie pendant cette période, mais sa réputation de metteur en scène parvient jusqu'à Budapest. Son nom 
est progressivement associé au mouvement "sécessionniste" qui se développe à Budapest et qui appelle à sortir du naturalisme, suivant l'exemple de la Wiener Sezession. Sa venue, en 1907, est ainsi annoncée par un article titré «Sortir du naturalisme ", par Lajos Roth. À cette époque, Reinhardt est non seulement perçu en Hongrie comme une figure totalement dissociée de Brahm, mais également comme le vecteur du dépassement des apports de ce dernier. De même que la figure de Brahm a cristallisé et incarné l'apport naturaliste étranger, la figure de Reinhardt condense et incarne la volonté d'en sortir.

7 À partir de 1910, les critiques remarquent et décrivent l'utilisation originale du son et des décors proposée par Reinhardt. En 1911, il présente pour la première fois un spectacle en arène, Edipe Roi. Si la critique est mitigée, le public est favorable et là encore, il annonce le succès qu'auront peu de temps après les représentations en plein air. Il continue à jouir d'un prestige considérable dans les années suivantes. Il revient en 1912 (et est alors longuement interviewé par plusieurs journaux), puis en 1914, où il ne parvient toutefois pas à faire accepter par le public les pièces de Strindberg qu'il présente, pas plus que Frühlings Erwachen (Le Réveil du Printemps)de Wedekind. De même, quand il revient en 1916, si les critiques sont bonnes, elles ne suscitent plus le même enthousiasme qu'avant: Staud explique cette baisse d'intérêt par un phénomène d'érosion, ainsi que par le fait que le théâtre hongrois, sous son influence, s'est progressivement rapproché de son travail. Reinhardt vient ensuite beaucoup moins à Budapest, du fait de la guerre puis des difficultés économiques qu'elle entraîne. Signalons cependant son passage en 1926. C'est en 1931 qu'il vient en Hongrie pour la dernière fois.

8 Si Reinhardt est d'abord confondu avec Brahm, il s'en détache rapidement pour devenir, à compter de 1903, la figure dominante. Les années de son véritable triomphe s'étendent de 1910 à 1917. Il n'est évidemment pas le seul moderniste à marquer la Hongrie durant cette période ; cependant, la fréquence de ses venues, la longévité et la relative unanimité de sa reconnaissance ainsi que le prestige associé à son nom lui offrent un statut à part. Il se dote ainsi progressivement d'une véritable aura en Hongrie : le poète et romancier Dezsö Kosztolányi parle notamment d'un public qui " écoute pieusement» et surtout «admire " ces Allemands, avec « un sentiment de fête ${ }^{4} »$. Un véritable culte se crée autour de sa personnalité auprès d'une partie du public : Reinhardt est pleinement intégré au système naissant de starification ${ }^{5}$ qui se développe autour des nouvelles institutions théâtrales budapestoises. Tandis que Brahm est considéré comme une grande figure du théâtre européen, Reinhardt, durant ces années, devient un membre à part entière de la vie théâtrale budapestoise.

\section{Reinhardt, figure centrale du théâtre hongrois}

9 En effet, la présence de Reinhardt à Budapest ne se limite pas aux déplacements des compagnies auxquelles il participe ou qu'il dirige. Il joue également un rôle crucial de formateur du personnel théatral hongrois, qu'il s'agisse des dramaturges, des acteurs

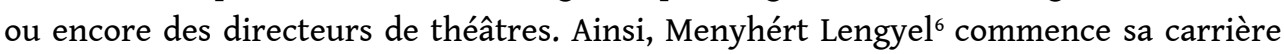
en obtenant une bourse de six mois à Berlin, en 1909, pour aller étudier chez Reinhardt. Son cas est emblématique : il précise en effet dans ses mémoires qu'à cette époque " tous les spécialistes et tous les directeurs de théâtre occidentaux venaient étudier chez lui $»^{7}$. Il retourne régulièrement à Berlin dans les années 1920, alors qu'il vit entre 
les États-Unis et l'Europe occidentale, et exprime durant ces années à plusieurs reprises son admiration pour Reinhardt, en qui il voit un « chef d'orchestre hors du commun ». De même, la plupart des grandes stars budapestoises de l'époque se forment dans une des troupes de Reinhardt. Citons l'exemple de Lili Darvas, vedette féminine hongroise de l'époque : celle-ci travaille avec Reinhardt de 1925 à 1938 et affirme à plusieurs reprises qu'elle a tout appris auprès de lui. Elle se plie à ses méthodes de travail rigoureuses, travaille son accent, et lui servira même d'assistante réalisatrice en 1943 à New York, pour la mise en scène de Sons and Soldiers d'Irving Shaw. Sári Fédak, autre grande star féminine de l'époque, apparaît elle aussi dans quelques productions de Reinhardt. Enfin, le cas d'Artúr Bárdos illustre le poids de Reinhardt dans la formation des directeurs de théâtre : à la tête pendant plusieurs années du Théâtre du Centre Ville ainsi que du cabaret de la Scène Moderne (Modern Színpad), il a d'abord travaillé à Berlin aux côtés de Reinhardt en tant qu'assistant.

10 A ce rôle de formation, s'ajoutent des phénomènes de collaboration directe. Molnár, dramaturge phare de l'époque ${ }^{8}$, évoque à plusieurs reprises dans ses mémoires ${ }^{9}$ les immenses réceptions de Reinhardt auquel il prenait très régulièrement part. Leurs rapports sont également professionnels. Molnár sert régulièrement d'interprète à Reinhardt durant ses séjours à Paris durant l'entre-deux-guerres. Après la guerre, alors qu'ils sont tous les deux expatriés aux États-Unis, Reinhardt rend régulièrement visite à Molnár à New York. Cette amitié s'appuie manifestement sur une forte estime réciproque. Reinhardt programme en effet plusieurs des pièces de Molnár en Allemagne : Olympia est par exemple joué à cent-neuf reprises à Berlin, avec Lili Darvas - la troisième et dernière femme de Molnár - dans le rôle titre.

11 Ces circulations et ces collaborations créent un climat de proximité qui brouille les frontières et conduit in fine à une quasi-naturalisation de Reinhardt. Ainsi, dans les années 1910 on lui invente de manière récurrente des origines hongroises, la légende se propageant selon laquelle il serait né dans une famille autrichienne d'origine hongroise, et comprendrait en fait parfaitement le hongrois.

\section{Le théâtre hongrois, théâtre reinhardtien ?}

La présence protéiforme de Reinhardt à Budapest, ainsi que le poids symbolique qui lui est alloué, l'imposent logiquement comme un vecteur fondamental du renouvellement du théâtre hongrois. Loin d'être marginal, son apport est manifeste et multiple, et fait de Reinhardt un acteur à part entière de la réflexion hongroise sur les formes du théâtre.

\section{Renouvellement des lieux de théâtre}

13 Un premier apport, clairement identifiable, réside en l'importation du modèle des Kammerspiele. Une première tentative a d'abord lieu par le biais des anciens membres de la compagnie moderniste Thália ${ }^{10}$ : après la dissolution de celle-ci, certains d'entre eux s'efforcent en effet de mettre en place en Hongrie des Kammerspiele sur le modèle reinhardtien, durant les années 1917-18. Ces tentatives, qui s'inscrivent dans un projet moderniste, ne parviennent pas à s'imposer : pas plus que la compagnie Thália à son époque, elles ne rencontrent l'adhésion du public. Le modèle s'implante néanmoins en 
Hongrie quelques années plus tard, au sein des grandes institutions, qui jouissent déjà d'un public et de structures établies. Est ainsi créé en 1924 le premier théâtre de chambre au Théâtre National, tandis que la même année, Arthur Bárdos inaugure au Théâtre du Centre Ville une scène petite et intime, rompant avec la tradition du théâtre monumental favorisé jusque là par cette institution.

Une seconde modalité d'apport concerne la renaissance du cabaret. L'époque de l'arrivée du Schall und Rauch coïncide en effet avec une période de redéfinition du cabaret à Budapest. Alors qu'il était dominé jusqu'à la fin des années 1890 par la tradition du cabaret en allemand, cette période s'achève en 1890 , date à laquelle le dernier cabaret en langue allemande ferme. Cette situation est le résultat d'une volonté politique de promotion d'une culture nationale; ainsi, à partir de 1895, les théâtres de variétés sont tenus de jouer au moins $50 \%$ de leur programme en hongrois. Cette législation n'est pas sans poser problème, puisque l'opinion commune veut que le hongrois, trop terne, se prête mal à un spectacle de ce type. Pour toutes ces raisons, le tournant du siècle est une période de crise, durant laquelle le genre est relativement délaissé. Le succès populaire et critique du Schall und Rauch ouvre alors la voie à une véritable renaissance. Les cabarets qui se développent à Budapest à compter de 1907, la Bonbonnière, le Modern Színpad [La Scène moderne] ou encore le Intim Színpad [La Scène Intime] sont largement et ouvertement inspirés de ce modèle. Ces lieux de divertissement développent une formule qui cherche à sortir des limites du cabaret traditionnel, au profit d'un aspect plus théâtral : ils programment aussi des pièces, et font venir des écrivains auquel ils demandent de rédiger de courts textes, qui sont lus en alternance avec des numéros plus traditionnels. Dans la lignée des conceptions de Reinhardt, la vocation de ces lieux est manifestement de remettre en cause et de dépasser la distinction entre théâtre populaire et théâtre élitiste: ces nouvelles formules, qui s'intègrent parfaitement au contexte budapestois de renouveau de l'amusement populaire, rencontrent un véritable succès.

\section{Transformations de la scénographie}

15 Au tournant du siècle, les premières transformations de la scène hongroise, comme partout en Europe, se font sous l'influence de l'esthétique naturaliste. À ce triomphe succède cependant une certaine lassitude, qui gagne aussi bien le public que les hommes de théâtre budapestois. Dans ses écrits, Hevesi ${ }^{11}$, directeur du Théâtre National, évoque la nécessité de montrer la vérité et de donner à celle-ci un cadre approprié. Il explique que les véritables intérieurs et les décors réalistes font une forte impression sur le public budapestois mais qu'ils ne font que masquer les vraies questions, liées à la nécessité de donner à voir le mouvement et de mettre en scène les évolutions durant le temps de la pièce. Il fait ainsi construire en 1927 une scène tournante au Théâtre National, explicitement sur le modèle de celles conçues par Reinhardt.

16 Hevesi souhaite sortir de la reproduction de la réalité, pour privilégier l'illusion et la suggestion: on retrouve chez lui les questionnements de la plupart des metteurs en scène européens, entre reconnaissance des apports du naturalisme et volonté de le dépasser. Reinhardt est à la fois partie prenante de ce mouvement et vecteur privilégié de la propagation de cette réflexion européenne en Hongrie $^{12}$. Son poids et son influence sont perceptibles dans la réflexion de Hevesi, marquée par une volonté de 
développer la dimension mystique de la représentation théâtrale, de recréer une communion entre le spectateur et les acteurs en renouant avec le théâtre antique. Il s'intéresse notamment au décor mouvant, à la lumière électrique - dans laquelle il voit la possibilité de créer une atmosphère et de modifier la décoration en modulant les éclairages - ou encore au gramophone, qui permet d'introduire des effets hors scène : ces innovations techniques sont mises au service d'un retour à la vocation originelle du théâtre.

\section{Acteurs et metteur en scène}

Lorsque Hevesi souligne dans ses écrits la montée en puissance du metteur en scène partout en Europe, il précise que la question se pose selon lui en Hongrie dans les mêmes termes qu'en Allemagne - plutôt qu'en France ou en Italie - parce que les acteurs y ont développé un style de jeu comparable. La remarque n'est pas dénuée de pertinence: si les réponses apportées divergent, on peut effectivement noter des correspondances significatives dans la manière dont ces questions sont abordées par Mór Ditrói, directeur du Théâtre Comique hongrois et principal inventeur d'un style national de mise en scène, et Max Reinhardt.

Sur sa conception du jeu de l'acteur, c'est incontestablement des travaux de Stanislavski que se rapproche Ditrói ${ }^{13}$ : il fonde en effet son travail sur la revalorisation du pathos, compris au sens propre comme mouvement de l'âme. Celui-ci serait la condition de la spontanéité ainsi que du naturel des gestes et mouvements sur scène. Il donne le primat à l'action physique des acteurs, à leurs déplacements sur scène, tout en précisant que « tout doit venir de l'intérieur. » Cette méthode est qualifiée par Mészöly, historien du théâtre hongrois, de "réalisme intérieur " ${ }^{14}$. En revanche, la proximité de Reinhardt est palpable dans l'élaboration des méthodes de travail. Ainsi, les répétitions sous la direction de Ditrói se déroulent en deux phases: une première phase d'observation, durant laquelle le metteur en scène laisse les acteurs s'exprimer librement, puis une seconde phase où il intervient. Reinhardt, dans une interview de 1915, parle lui aussi de phases: le metteur en scène doit d'abord laisser les acteurs jouer à leur manière, afin de pouvoir connaître parfaitement sa matière. Les deux hommes se retrouvent également dans l'importance attribuée à la distribution. Le rôle du metteur en scène, chez Ditrói consiste avant tout à connaître sa matière actorale, afin de pouvoir répartir au mieux les rôles; si Reinhardt accorde évidemment un rôle beaucoup plus large au metteur au scène, il insiste lui aussi sur l'importance de la distribution : dans sa conception, celle-ci serait une «partition", écrite pour un acteur en particulier, et comprenant déjà une «vision optique et acoustique " préalable à toute répétition.

19 À l'étape suivante, en revanche, leurs conceptions divergent clairement. Reinhardt considère que le metteur en scène doit ensuite faire émerger «l'esprit» de la représentation et voit dans le metteur en scène une puissance positive, chargée de " créer les conditions dans lesquelles tout ce que l'auteur a indiqué devient possible ${ }^{15}$ ». Ditrói poursuit au contraire un idéal de transparence : le metteur en scène doit être " anonyme ", et le spectateur ne doit "même pas se rendre compte qu'il y a une mise en scène ». Il s'agirait simplement d'ajuster la scène " au point de vue correct », c'est-àdire d'adopter celui du spectateur, et de veiller à ce que les règles du naturel soient respectées. 

laisse des marques sur le théâtre hongrois : plus qu'un apport clairement identifié ou d'une filiation explicite, il s'agit en effet plutôt de traces fragmentées, diffuses, qui parsèment la création théâtrale budapestoise et la marquent à divers niveaux. Les conceptions de Reinhardt sont à la fois omniprésentes et difficiles à circonscrire : il plane sur la vie théâtrale durant ces années comme un référent, une source d'inspiration et une figure tutélaire.

La place centrale occupée par Reinhardt dans les phénomènes d'échanges et de circulations théâtrales entre Vienne, Budapest et Berlin, la fréquence de ses passages à Budapest ainsi que le développement d'une aura attachée à son nom font de lui une figure majeure de la Hongrie du tournant du siècle. Il participe à plein de la création théâtrale hongroise, à la fois en tant qu'inspirateur, formateur, interlocuteur et collaborateur direct. S'il prend d'abord la succession de Brahm dans le rôle d'importateur d'un renouveau théâtral, il acquiert ensuite une importance inédite dans le champ hongrois. D'invité prestigieux, il devient partie intégrante de la vie théâtrale. La confusion autour de sa nationalité est loin d'être anecdotique: durant ces années, Reinhardt est véritablement perçu par les hommes de théâtre hongrois comme étant l'un des leurs.

\section{BIBLIOGRAPHIE}

Yves Chevrel, Le Naturalisme : étude d'un mouvement littéraire international, PUF, 1993.

Tamás Gajdo (dir.), Magyar szinház történet 1873-1920, (Histoire du théâtre hongrois 1873-1920), Budapest, Magyar Könyklub, 2001.

Miklós Gyárfás (dir.), Élö dramaturgia, (Dramaturgie vivante), Budapest, Magvetö Kiadó, 1963.

Sándor Hevesi, Az elöadás, a színjátszás, a rendezés müveszete, (L'art de la représentation, du jeu et de la mise en scène), Budapest, Gondolat kiadó, 1965.

Deszö Kosztolányi, Szinházi esték, (Soirs de théâtre), Budapest, Szépirodalmi könyvkiadó, 1978.

Ményhért Lengyel, Életem könyve, (Le Livre de ma vie), Budapest, Gondolat Kiadó, 1987.

Bálint Magyar, A Vígszínház története, (Histoire du Théâtre Comique), Budapest, Szépirodalmi Könyvkiadó, 1979.

Ferenc Molnár, Companion in exile. Notes for an autobiography, New York, Gaer Associates, 1950.

Tibor Mészoly, Színház a század küszobén-Ditrói Mór és a Vígszinház stilusforradalma, (Théâtre autournant du siècle: Mór Ditrói et la révolution stylistique du Théâtre Comique), Budapest, Múzsák Közmüvelödési Kiadó, 1986.

Endre Nagy, A Kabaré regénye, (Le Roman des cabarets), Budapest, Palatinus Könyvek, 2000.

György Székely, A Nemzeti Szinház, (Le Théâtre National), Gondolat, Budapest, 1965.

Germanica, 43 | 2008 
Margit Török (dir.), Max Reinhardt Szinháza, (Le Théâtre de Max Reinhardt), Budapest, Országos Szinháztörténeti Múzeum és Intézet, 1994.

\section{NOTES}

1. Voir à ce sujet notamment Yves Chevrel, Le Naturalisme: étude d'un mouvement littéraire international, Paris, PUF, 1993.

2. Voir notamment « Max Reinhardt Magyarországon » (« Max Reinhardt en Hongrie »), in Margit Török (dir), Max Reinhardt Szinháza (Le Théâtre de Max Reinhardt), Budapest, Országos Szinháztörténeti Múzeum és Intézet, 1994, et STAUD Géza, «Külföldi hatások a magyar szinjátszásban, rendezésben és dramaturgiában » («Influences étrangères sur le jeu, la mise en scène et la dramaturgie hongrois »), in Gyárfás Miklós (dir.), Elö dramaturgia (Dramaturgie vivante), Budapest, Magvetö Kiadó, 1963.

3. Celui-ci trouve d'autant plus d'écho qu'un des grands classiques du théâtre hongrois, $A z$ ember tragédiája (La Tragédie de l'Homme) - adapté en français par Jean Rousselot, Budapest, Corvina, 1966 - d'Imre Madách, long poème dramatique, retrace les grandes étapes de l'histoire de l'humanité. Ce drame sera interprété dans le style Meininger pendant de très nombreuses années.

4. Voir Dezsö Kosztolányi, Szinházi esték (Soirs de théâtre), Budapest, Szépirodalmi Könyvkiadó, 1978.

5. Ces années marquent notamment l'apparition des fan-clubs d'actrices, comme le «Club Katalin Karády » (Karády Katalin Klub, KKK).

6. Menyhért Lengyel (1880-1974) est une des grandes figures du théâtre populaire hongrois des années 1910 à 1930. Il est notamment l'auteur du Grand Souverain, de Typhon d'Ange; il écrit également le livret du Mandarin Merveilleux, mis en musique par Bartók. Il s'installe à Hollywood dans les années 1940, où il commence une seconde carrière de scénariste, essentiellement marquée par sa collaboration avec Lubitsch : il est ainsi l'auteur des scénarios de Ninotschka et de To Be or Not to Be.

7. Voir Menyhért Lengyel, Életem könyve (Le Livre de ma vie), Budapest, Gondolat Kiadó, 1987.

8. Ferenc Molnár (1878-1952) est un dramaturge hongrois dont les œuvres connaissent un succès colossal, tant en Hongrie que dans la plupart des capitales européennes ainsi qu'à Broadway, du milieu des années 1900 jusqu'à la fin des années 1930. Parmi ses plus grands succès, citons Le Loup, Liliom, Le Garde ou encore Olympia. Si l'essentiel de sa production est théâtrale, son roman Les Gars de la rue Paul jouit également d'une large reconnaissance populaire.

9. Ferenc Molnár, Companion in exile. Notes for an autobiography, New York, Gaer Associates, 1950.

10. La compagnie Thália fonctionne de 1904 à 1908 et se modèle sur le Théâtre Libre d'Antoine, ou encore sur la Freie Bühne. Sa vocation consiste avant tout à importer des pièces étrangères, notamment naturalistes, s'inscrivant dans un courant visant à promouvoir l'art et la philosophie modernes en Hongrie.

11. Sándor Hevesi, Az elöadás, a színjátszás, a rendezés müvészete (L'Art de la représentation, du jeu et de la mise en scène), Budapest, Gondolat Kiadó, 1965.

12. Il n'est cependant pas le seul: Hevesi entretient notamment une correspondance fournie et suivie avec Gordon Craig, qui alimente en permanence sa réflexion personnelle.

13. Au point qu'on parle fréquemment de lui comme du Stanislavski hongrois, certains critiques n'hésitant pas à affirmer que Ditrói a sans doute été une source d'inspiration pour le travail de Stanislavski. 
14. Tibor Mészöly, Színház a század küszöbén-Ditrói Mór és a Vígszinház stillusforradalma, (Théâtre au tournant du siècle: Mór Ditrói et la révolution stylistique du Théâtre Comique), Budapest, Múzsák Közmüvelödési Kiadó, 1986.

15. In «A rendezöpéldány » («L'exemplaire du metteur en scène »), sélection d'écrits de Max Reinhardt, in Margit Török (dir.), Max Reinhardt Szinháza, (Le Théâtre de Max Reinhardt), Budapest, Országos Szinháztörténeti Múzeum és Intézet, 1994.

\section{RÉSUMÉS}

Si le théâtre hongrois a toujours entretenu des liens étroits avec l'aire germanophone, la reconfiguration des industries du spectacle, au tournant du siècle, intensifie considérablement les phénomènes d'échanges et de correspondances. Max Reinhardt, d'abord dans le sillage d'Otto Brahm puis de manière indépendante, s'impose ainsi comme un vecteur essentiel du renouveau théâtral en Hongrie. Son apport est à l'image de sa présence, fragmenté et protéiforme : invité récurrent, figure tutélaire, instance de formation, il apparaît comme une source d'inspiration à la fois diffuse et omniprésente. Sa marque se lit tant dans la volonté de renouveler les lieux de théâtre ou de repenser la scénographie que dans la manière dont la réflexion sur les rapports entre acteur et metteur en scène se structure. Durant les années 1910, son aura en Hongrie est telle qu'elle conduit à une quasi-naturalisation: plus qu'un invité prestigieux, Reinhardt est perçu et célébré comme un membre à part entière de la communauté théâtrale hongroise.

Das ungarische Theater ist seit jeher stark von der deutschsprachigen Theaterszene beeinflußt worden; die wechselseitige Beeinflussung wurde noch durch den sich in der Kulturindustrie vollziehenden Wandel um 1900 erheblich verstärkt. Max Reinhardt tritt hierbei zuerst im Kielwasser von Otto Brahm, dann im eigenen Namen als Grunderneuerer des Theaters in Ungarn hervor. Sein Beitrag gleicht seinem Wirken, sporadisch und mannigfaltig zugleich: als ständiger Gast, als Vater- und Lehrerfigur stellt er ein nachzuahmendes, gleichzeitig vages und allgegenwärtiges Vorbild dar, das manchen beflügelt. Sein Einfluß ist sowohl im Bestreben, die Theaterbühne zu erneuern oder die Szenographie neu zu denken, als auch in der Art und Weise, wie die Beziehungen zwischen Schauspieler und Regisseur sich neu gestalten, klar abzulesen. In den 1910er Jahren ist seine Ausstrahlung in Ungarn so groß, dass man ihn vereinnahmt und quasi eingebürgert hat: mehr als nur Vorzeigegast, wird Reinhardt als der ungarischen Theaterszene voll angehörig betrachtet und gefeiert.

\section{INDEX}

Mots-clés : Budapest

\section{AUTEURS}

\section{KATALIN PÓR}

Université Paul Verlaine, Metz 